\title{
COMPOSIÇÃO CORPORAL E ESTADO MENOPAUSAL EM MULHERES COM CÂNCER DE MAMA: UMA REVISÃO DA LITERATURA
}

\section{BODY COMPOSITION AND MENOPAUSAL STATUS IN WOMEN WITH BREAST CANCER: A LITERATURE REVIEW}

\author{
Taiara Scopel Poltronieri ${ }^{\mathrm{a}^{*}}$, Rafaela Liberali $^{\mathrm{a}^{*}}$ \\ taiarapoltronieri@hotmail.com ${ }^{\mathrm{a}}$ \\ Universidade Municipal de São Caetano do Sul (USCS), São Caetano do Sul-SP, Brasil ${ }^{*}$
}

Data do recebimento do artigo: 23/05/2018

Data do aceite: $02 / 07 / 2018$

\begin{abstract}
RESUMO:
Introdução: A composição corporal de mulheres diagnosticadas com câncer de mama está propensa a alterações por fatores como idade, estado menopausal, existência e extensão da doença e tratamento antineoplásico. Este cenário é de extrema importância, já que pode vir a interferir também no resultado clínico destas. Objetivo: Avaliar a relação entre composição corporal, estado menopausal e possíveis desfechos clínicos em mulheres com câncer de mama. Materiais e métodos: Revisão integrativa da literatura realizada nas bases de dados PubMed, Scielo e Bireme. Buscaram-se artigos publicados até 2018 em português, inglês ou espanhol. Os critérios de inclusão foram: estudos longitudinais, conduzidos em mulheres na prée pós-menopausa, após diagnóstico de câncer de mama, em qualquer estágio e que avaliaram a relação entre estado menopausal e composição corporal. Avaliou-se a qualidade metodológica dos selecionados. Resultados: Seis estudos foram elegíveis, dentre os quais, somente quatro encontraram associação entre alterações na composição corporal e o estado menopausal nesta população. Destes, um mostrou esta relação entre pacientes na pré-menopausa, enquanto que em outros dois, esta foi estatisticamente significativa na pós-menopausa, sendo a sobrevida livre de doença a longo prazo pior nas mulheres pós-menopáusicas com alta quantidade de gordura visceral. Outro, relatou que as alterações foram similares entre ambos os estados menopausais. Conclusão: A presente revisão inova em abordar este assunto, no entanto, mostrou falta de evidências consistentes em relação aos tópicos avaliados. Ademais, visto serem escassos os trabalhos comparando a relação destes com desfechos clínicos entre esses indivíduos, mais estudos são necessários para esclarecer este contexto.
\end{abstract}

Palavras Chaves: Composição corporal; pré-menopausa; pós-menopausa; câncer de mama.

\section{ABSTRACT}

Introduction: Body composition in breast cancer diagnosed women is inclined to alterations by factors like age, menopause status, disease exist and extension and antineoplastic treatment. This background is extremely important, because can interfere also on their clinical results. Objective: To evaluate the relationship between body composition, menopausal status and possible clinical outcomes in breast cancer women. Materials and methods: Integrative review of literature performed on PubMed, Scielo and Bireme databases. It was looked for articles published until 2018 in Portuguese, English or Spanish. The inclusion criteria were: longitudinal studies, conducted in pre- and postmenopausal women, after breast cancer diagnoses, in any stage and which evaluated the relationship between menopausal status and body composition. The methodological quality of the selected papers was accessed. Results: Six studies were eligible, among whom, just four found association between body composition alterations and menopausal status at this population. Of these, one showed this relation between premenopausal patients, while in two others, this was statistically significant at postmenopausal, being the long-term disease-free survival worse on postmenopausal women with high visceral fat quantity. Another study, reported that the changes were similar between both menopausal status. Conclusion: The present review innovate in approaching this subject, however, it showed lack
} 
of consistence evidences with regard to the evaluated topics. Furthermore, once the researches comparing the relationship of these with clinical outcomes on these individuals are scarce, more studies are necessary to clarify this context.

Keywords: Body composition; premenopause; postmenopause; breast neoplasms. 


\section{Introdução}

O mecanismo carcinogênico é caracterizado pela presença de tumor maligno, com rápido crescimento celular e potencial para atacar e se espalhar para outras partes do corpo ${ }^{1}$. O câncer de mama (CM) é a neoplasia de maior incidência e a principal causa de morte por câncer entre mulheres ao redor do mundo ${ }^{2,3}$. Para 2018-2019, no Brasil, o Instituto Nacional de Câncer (INCA) estima 59.700 casos novos desta malignidade para cada ano, números aumentados em relação ao biênio anterior ${ }^{4}$. Trata-se de um grupo de doenças distintas, o qual apresenta diferentes manifestações, tratamentos e prognósticos ${ }^{5,6}$.

A etiologia desta patologia é complexa, já que seu desenvolvimento está relacionado à genética, alterações hormonais e ao estilo de vida e meio ambiente, como obesidade, elevado percentual de gordura corporal, hábitos alimentares negativos, sedentarismo, ocupações estressantes, entre outros $^{7-16}$. Diversas abordagens terapêuticas estão disponíveis, as quais denominam-se terapias locais, como métodos cirúrgicos e radiação, e sistêmicas, como quimioterapia, terapia endócrina e terapia alvo ${ }^{17}$.

De acordo com a literatura, o estado nutricional dos pacientes oncológicos, principalmente no que se refere à composição corporal (quantidade de tecido muscular e adiposo) pode ser alterado por uma complexa interação entre fatores, como a própria idade, a existência e extensão da doença e as alterações oriundas do tratamento antineoplásico. Levando com isso, à redução da massa magra, com manutenção ou aumento de gordura, quadro visto com frequência em pacientes com $\mathrm{CM}^{18-21}$. Entre as mulheres diagnosticadas com esta neoplasia, tem-se ainda, o estado menopausal como variável de extrema importância que pode vir a interferir tanto na composição corporal, como no próprio resultado clínico, uma vez que, a produção de estrogênio, hormônio envolvido na progressão tumoral, sofre alterações nos diferentes estágios menopausais ${ }^{22-24}$.

Estas alterações nos tecidos corporais estão diretamente relacionadas à manutenção da inflamação crônica, recidiva de doença, piora da capacidade funcional e da qualidade de vida, maior toxicidade terapêutica e desordens metabólicas, aumento de morbidades e mortalidade, entre outros ${ }^{18,20,25-27}$. Desta forma, parece que o controle do peso corporal dentro do adequado, com especial vigilância da composição corporal, poderia trazer benefícios clínicos imprescindíveis a estes indivíduos ${ }^{24,25}$.

Neste contexto, o objetivo deste estudo foi avaliar, através de revisão integrativa da literatura, a relação entre composição corporal, estado menopausal e possíveis desfechos clínicos em mulheres com CM.

\section{Metodologia}

\section{TIPO DE PESQUISA}

A presente revisão integrativa foi desenvolvida de acordo com o que é determinado pela diretriz PRISMA (Preferred Reporting Items for Systematic Reviews and Meta-Analyses) ${ }^{28}$, visando sistematizar sua construção e, com isto, conferindo maior qualidade ao estudo.

\section{ESTRATÉGIA DE BUSCA}

Executou-se entre fevereiro e março de 2018, uma busca sistematizada nas bases de dados: U.S. National Library of Medicine (PubMed), Scientific Electronic Library Online (Scielo) e Biblioteca Virtual em Saúde (Bireme), tendo sido os termos empregados nesta, selecionados de acordo o PubMed's Medical Subject Headings (MeSH). O quadro 1 mostra um exemplo completo de estratégia utilizada. O processo foi realizado em língua inglesa, sendo composto por três blocos conceituais: o primeiro com termos relacionados ao CM (breast cancer, breast neoplasm, breast tumor, breast carcinoma); o segundo com termos referentes à composição corporal (body composition, body compositions, adipose tissue, body fat, skeletal muscle); e o último condizente a desfechos clínicos (clinical outcome ${ }^{*}$ patient outcome assessment, prognoses, prognosis, survival). Os operadores booleanos "OR" e "AND" foram utilizados para a combinação dos descritores dentro de cada bloco e para combinar os blocos entre si, respectivamente, com truncagem dos termos quando necessário. Consideraram-se os estudos publicados até março de 2018 em língua portuguesa, inglesa ou espanhola. Adicionalmente, foi feita uma busca manual por possíveis materiais que pudessem constar na lista de referências dos artigos selecionados. 
Quadro 1 - Exemplo de estratégia de busca utilizada.

((((()breast cancer) OR breast neoplasm) OR breast tumor) $O R$ breast carcinoma)) AND (((((body composition) OR body compositions) OR adipose tissue) OR body fat) OR skeletal muscle)) AND ((((clinical outcome $\left.{ }^{*}\right)$ OR patient outcome assessment $\left.{ }^{*}\right)$ OR prognoses) OR prognosis) OR survival).

\section{CRITÉRIOS DE ELEGIBILIDADE}

Os critérios de inclusão foram: (1) estudos conduzidos em humanos; (2) de amostra exclusivamente feminina; (3) abordando somente pacientes com CM após o diagnóstico em qualquer estágio da doença; (4) na pré- e pós-menopausa; (5) que avaliaram a relação entre estado menopausal e composição corporal; (6) com desenho longitudinal. Foram excluídos aqueles que utilizaram apenas peso ou índice de massa corporal ou circunferências de cintura e quadril para avaliação antropométrica ou os que tinham como desfechos o risco para desenvolvimento de CM.

\section{SELEÇÃO DOS ESTUDOS}

Os artigos foram selecionados inicialmente com base na leitura do título, seguida pela avaliação dos resumos. A partir dos resumos selecionados, os artigos completos foram revisados de forma criteriosa, levando em consideração os critérios de inclusão e exclusão do presente estudo.

\section{EXTRAÇÃO DE DADOS}

Foi desenvolvido um formulário padrão com os itens a serem considerados durante a coleta de dados a partir das publicações selecionadas. Incluíram-se nesta ferramenta informações quanto aos autores, ano de publicação, local, desenho do estudo, objetivos, critérios de inclusão/exclusão, características (idade, estado menopausal etc.) e tamanho da amostra, metodologia de intervenção (quando apropriado) e acompanhamento, métodos e frequência da avaliação da composição corporal, desfechos clínicos analisados, possíveis fontes de viés, análise estatística empregada, ajuste de variáveis, limitações, principais conclusões, entre outros.

\section{AVALIAÇÃO DA QUALIDADE}

Em virtude de terem sido elegíveis para esta revisão somente artigos de desenho longitudinal, a avaliação da qualidade destes foi feita através da escala de Newcastle-Ottawa (NOS), que avalia a qualidade de estudos não-randomizados, do Ottawa Hospital Research Institute ${ }^{29}$ e, da ferramenta ROBINS-I, que avalia o risco de viés de estudos de intervenção não randomizados desenvolvida pelo Cochrane Group ${ }^{30}$. A NOS é dividida em três categorias, sendo cada uma delas composta por itens condizentes a processos de desenvolvimento de artigos científicos longitudinais. Para avaliação dos estudos, cada item das categorias seleção e desfecho pode receber uma estrela, enquanto o item que compõem a categoria comparabilidade pode receber até duas estrelas ${ }^{29,31}$. Já a ROBINS-I é dividida em sete domínios, os dois primeiros relacionados a questões prévias ao início das intervenções (confusão e seleção dos participantes do estudo). O terceiro domínio aborda fatores relativos às próprias intervenções e, os outros quatro abordam questões posteriores ao início destas (presença de vieses devido a desvios das intervenções pretendidas, dados faltantes, mensuração de resultados e seleção dos resultados apresentados). De acordo com a análise destes tópicos, o risco de viés do estudo avaliado pode ser classificado como baixo, moderado, grave ou crítico ${ }^{30,32}$.

\section{APRESENTAÇÃO DOS RESULTADOS}

Devido à heterogeneidade metodológica entre os estudos incluídos, considerou-se a abordagem narrativa como a melhor metodologia para síntese dos resultados.

\section{RESULTADOS}

O fluxograma mostrando o processo de seleção é apresentado na figura 1. Encontrou-se um total de 1404 artigos, dos quais 1294 foram excluídos por não condizerem com o objeto desta revisão e 11 por se repetirem, sendo selecionados 99 estudos para análise mais criteriosa. Destes, 40 foram excluídos por não preencherem os critérios de inclusão e os demais 59 foram submetidos à avaliação completa. Ao final, seis estudos foram elegíveis para esta análise. 
Figura 1: Fluxograma do processo de seleção dos estudos avaliados.

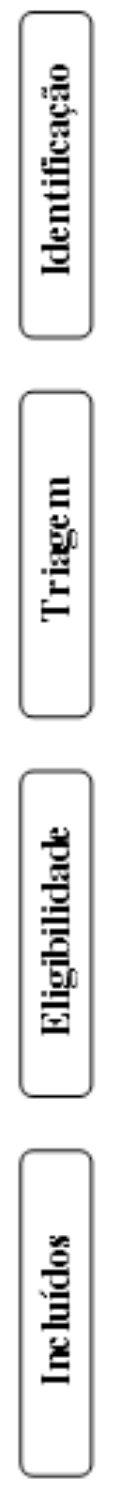

Estudos identificados através da busca eletrônica nas bases de dados: PubMed $(n=1029)$, Bireme $(n=364)$ e SciELO $(n=11)$.

$(n=1393)$.
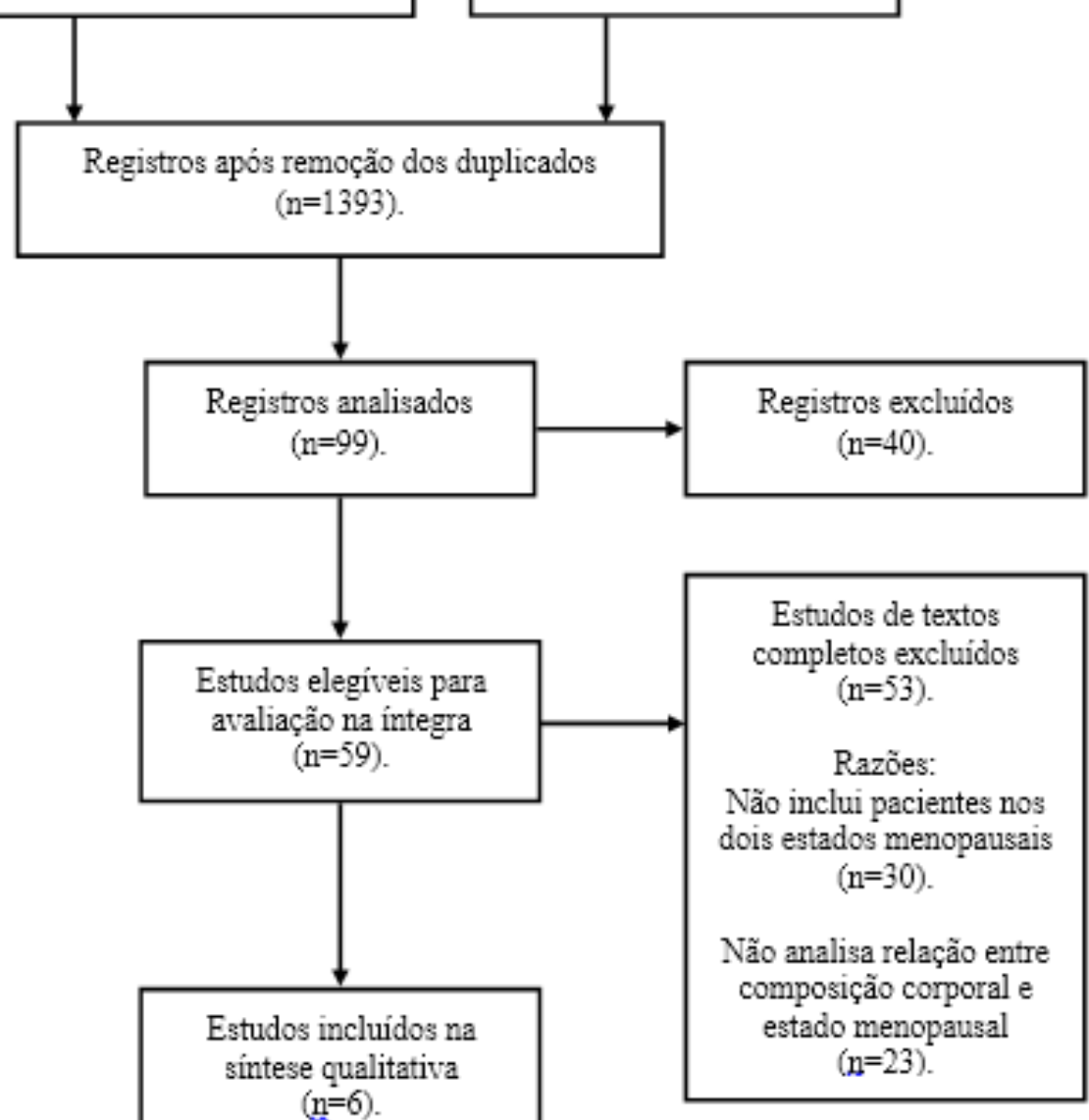

As principais características de cada estudo e resultados das associações encontradas, assim como a avaliação da qualidade metodológica e as variáveis de controle utilizadas estão descritos na Tabela 1 (Anexo). Todos os seis artigos incluídos abordaram pacientes do sexo feminino, na pré- e pós-menopausa, diagnosticadas com $\mathrm{CM}$ e tiveram desenho longitudinal, de caráter observacional ${ }^{33-35,37,38}$ e quase experimental ${ }^{36}$. Sendo um retrospectivo ${ }^{35}$ e os demais prospectivos ${ }^{33,34,36}$, incluindo duas coortes ${ }^{37,38}$. Apenas um deles apresentou grupo controle ${ }^{33}$. No total, o tamanho das amostras variou de 40 a 471 pacientes $^{33-38}$.
Em relação à idade dos indivíduos investigados, quatro artigos forneceram essa informação na forma de média, sendo 48,2 anos ${ }^{34}, 54$ anos $^{35}$, $54,32 \operatorname{anos}^{33}$ e 56,3 anos ${ }^{38}$. Já outro, informou a variação da faixa etária que foi de 32-61 anos ${ }^{36}$ e, por fim, Villasenor et al. apresentaram a mediana de idade do grupo classificado como não sarcopênico e do sarcopênico, sendo 56 anos e 63,9 anos, respectivamente ${ }^{37}$.

Quanto ao estado menopausal, em cinco estudos o maior percentual foi de pacientes na pós-menopausa, sendo $52,5 \%{ }^{36}, 57 \%^{35}, 66,9 \%$ (apresentou missing $)^{37}, 69 \%^{38}$ e $73,6 \%{ }^{33}$. Por outro lado, 
no estudo de Dieli et al., a maior parte das mulheres encontrava-se na pré-menopausa $(53 \%)^{34}$. Somente Cisneros et al. informaram que consideraram pós-menopausa como a ausência de menstruação por mais de um ano ${ }^{36}$, os demais estudos não citaram essa questão $0^{33-35,37,38}$. Ainda, em três trabalhos foi possível verificar o momento condizente ao qual essa classificação do estado menopausal aconteceu, sendo, o presente após a radioterapia adjuvante ${ }^{33}$, o apresentado no momento do diagnóstico ${ }^{34}$ e na entrada do estudo, ou seja, após um ano do diagnóstico ${ }^{38}$. Os trabalhos restantes não mencionaram essa informação ${ }^{35-37}$.

Relativo aos estadiamentos apresentados, cinco estudos investigaram pacientes com câncer estadio variando entre I-III ${ }^{33,34,36-38}$, sendo que Irwin et al. abordaram também aquelas com neoplasia in situ $^{38}$. Já Iwase et al., incluíram mulheres com CM estadio entre II-IV, este último indicando pacientes com doença avançada ${ }^{35}$. No que diz respeito ao momento clínico dos indivíduos investigados, um estudo avaliou pacientes antes do início de quimioterapia neoadjuvante ${ }^{36}$. Dieli et al., abordaram aquelas com diagnóstico recente de $\mathrm{CM}$, que estavam realizando quimioterapia adjuvante ou neoadjuvante ${ }^{34}$. Já Iwase et al. avaliaram mulheres submetidas à cirurgia após realização de somente quimioterapia neoadjuvante ${ }^{35}$. Por fim, três estudos abordaram pacientes previamente submetidas a tratamento oncológico, o qual poderia incluir cirurgia, quimioterapia e radioterapia ${ }^{33,37,38}$. Ademais, nas pesquisas de Akiol et al. e Irwin et al., as pacientes estavam fazendo uso de hormonioterapia no momento do estudo ${ }^{33,38}$.

Foram abordados três métodos de avaliação da composição corporal, sendo que 50\% dos estudos utilizaram densitometria por dupla emissão de raios- $\mathrm{X}^{36-38}$, dois lançaram mão de impedância bioelétrica $^{33,34}$ e um usou tomografia computadorizada $^{35}$. No que tange à frequência dessa avaliação, quatro estudos a realizaram em dois momentos diferentes, dos quais um aconteceu na entrada do estudo (basal) e as demais após o tratamento ou na finalização do seguimento ${ }^{33,34,36,38}$. Os outros dois avaliaram a composição corporal apenas uma vez, em um deles foi realizada na entrada do estudo $^{37}$ e no outro, antes do início da investigação ${ }^{35}$.

O tempo de seguimento dos trabalhos variou entre 6 meses $^{36}$, um ano ${ }^{33}$ e 3 anos $^{34,38}$. Nas coortes, uma teve média de 9,2 anos de acompanhamento $^{37}$ e a outra, média de 1638 dias de sobrevida ${ }^{35}$.

$\mathrm{O}$ uso de controles nas análises foi visto em quatro estudos, alguns autores realizaram ajustes em todos os modelos ${ }^{34,37}$ e outros controlaram somente os que avaliavam a composição corpo$\mathrm{ral}^{36,38}$, sendo que grande parte destes utilizaram variáveis semelhantes. A idade foi ajustada por todos os quatro ${ }^{34,36-38}$, raça/etnia e tratamento em três ${ }^{34,37,38}$, estado menopausal e estadiamento também em três ${ }^{36-38}$ e local do estudo, uso de hormonioterapia e atividade física em dois ${ }^{37,38}$. Villasenor et al. e Irwin et al., realizaram ainda, ajustes para outras variáveis não abordadas pelos demais ${ }^{37,38}$. Por fim, os outros dois artigos não informam se houveram controles de análises ${ }^{33,35}$.

No tocante à avaliação da qualidade metodológica, apenas um artigo obteve o escore máximo de seis pontos conforme a NOS ${ }^{34}$, outros dois receberam cinco $^{37,38}$ e os demais computaram três pontos $^{33,35}$. Por sua vez, Cisneros et al., foi avaliado conforme a ROBINS-I, devido ao seu desenho quase experimental, e foi classificado com risco moderado de viés ${ }^{36}$.

Considerando os seis estudos incluídos, quatro encontraram associação entre estado menopausal e composição corporal ${ }^{33,35-37}$. Cisneros et al. observaram um aumento de em média $2,3 \mathrm{~kg}$ de tecido adiposo após a quimioterapia em pacientes na pré-menoupausa $(\mathrm{p}=0,001)$, achado não significativo entre aquelas na pós-menopausa $(p=0,227)^{36}$. Por outro lado, Iwase et al. identificaram aumento significativo dos indicadores de gordura corporal após a menopausa, enquanto que o indicador de massa magra diminuiu ( $p<0,05$ em ambos os casos). Da mesma forma em que a razão entre gordura visceral e subcutânea aumentou $(p<0,05)$ e a sobrevida livre de doença a longo prazo foi significativamente pior em pacientes na pós-menopausa com alta quantidade de gordura visceral em comparação com aqueles com baixa $(\mathrm{p}<0,05)^{35}$. Os resultados de Villasenor et al., também mostraram relação com a pós-menopausa, no qual a sarcopenia foi mais comum em mulheres nesta condição menopausal $(\mathrm{p}=0,001)^{37}$. Akiol et al. citaram somente que as alterações na composição corporal foram similares entre o grupo na pré-menopausa e o na pós-menopausa, porém não fornecem os resul- 
tados numéricos e nem a relação entre os achados de cada grupo ${ }^{33}$. Os outros dois estudos não encontraram qualquer associação entre estado menopausal e composição corporal nas pacientes avaliadas ${ }^{34,38}$.

\section{Discussão}

Este artigo sumarizou os achados literários acerca do estado menopausal e da composição corporal em pacientes do sexo feminino com CM. Tópico complexo, devido à sua provável etiologia multicausal, já que o aumento da adiposidade em relação à massa magra pode ter como moderadores, principalmente, a idade, o estado menopausal, o tratamento oncológico (em especial terapia hormonal e quimioterapia) e a duração dele, atuando de forma isolada ou interagindo entre $\mathrm{si}^{39-43}$. Por conseguinte, a compreensão destes mecanismos e suas implicações à saúde neste cenário são de grande relevância, posto que estas alterações hormonais e corporais são comumente encontradas nestas pacientes e, por estarem possivelmente associadas a desfechos clínicos desta população ${ }^{39,44}$.

Dos artigos incluídos nesta revisão que encontraram associação entre o estado menopausal e a composição corporal, um identificou aumento de tecido adiposo após tratamento sistêmico nas mulheres pré-menoupáusicas ${ }^{36}$, enquanto que outros dois mostraram maior quantidade de gordura do que massa magra em indivíduos na pós-menopausa $^{35,37}$. Já Akiol et al. relataram que as alterações foram similares entre o grupo na pré- e o na pós-menopausa, porém não forneceram subsídios quantitativos para avaliação dessa informação ${ }^{33}$.

De forma sucinta, o processo fisiológico relacionando estado menopausal com alterações nos tecidos corporais dá-se da seguinte forma: na pré-menopausa os ovários são as principais fontes de produção de estrogênio ${ }^{22-24}$. Já após a menopausa, ocorre redução da função ovariana e o tecido adiposo passa a ser fonte extragonadal deste hormônio, acrescido da sua capacidade de converter androgênio em estrogênio pela enzima aromata$\mathrm{se}^{23}$. Por tanto, mulheres com maior quantidade de gordura corporal na pós-menopausa tendem a ter maiores níveis circulantes de estrogênio, estrona e estradiol (hormônio semelhante ao estrogênio), paralelo a menores níveis séricos de globulina ligadora de hormônios sexuais que aquelas na pré-menopausa. Em níveis normais, esta glicoproteína se liga ao estradiol e inibe-o, ficando esta função comprometida se seus níveis estiverem inadequados ${ }^{22}$.

Similarmente aos achados dos estudos avaliados aqui, investigações com pacientes na pré-menopausa encontraram aumento especialmente na gordura do tronco, e tendências para diminuição da massa magra no primeiro ano após o tratamento quimioterápico ${ }^{40,45}$. Uma vez que, $50-70 \%$ das pacientes pré-menopáusicas tendem a desenvolver insuficiência ovariana induzida por quimioterapia ${ }^{46}$, é preciso considerar a possibilidade de que essas mudanças possam resultar não apenas do tratamento oncológico sistêmico diretamente, mas também da imersão súbita na menopausa induzido por ele e do próprio processo natural associado à idade ${ }^{39,47}$.

Em paralelo, evidências suportam a hipótese de que o declínio nos níveis de estrogênio procedentes da menopausa, atua na perda de massa muscular e na redistribuição da gordura subcutânea para a área visceral ${ }^{40,48}$. Porém, o acúmulo de massa gorda nessa região é mais deletério do que na região subcutânea, pois aumenta a entrega de ácidos graxos livres na circulação portal ${ }^{49}$. Observou-se que o aumento de gordura visceral durante a transição menopausal foi associado à produção de adipocinas inflamatórias, relacionando-se à resistência à insulina, diabetes mellitus tipo 2 , hipertensão, hiperlipidemia e doenças cardiovasculares $^{50,51}$. Além da redução da quantidade de massa magra, os estudos também tratam de mudanças nas características do tecido muscular durante a menopausa, mostrando que mulheres na pós-menopausa tinham duas vezes mais gordura intramuscular em comparação com as mais jovens ${ }^{52}$.

Apenas um entre os estudos analisados pelo presente trabalho mostrou associação entre o estado menopausal, composição corporal e desfecho clínico. Neste, a sobrevida livre de doença a longo prazo foi significativamente pior em pacientes na pós-menopausa com alta quantidade de gordura visceral do que naquelas com baixa quantia ${ }^{35}$. Achado que condiz com a literatura, já que, como 
visto acima, a produção de estrogênio tende a estar aumentada quando há elevada presença de tecido gorduroso e, pelo fato deste hormônio estar envolvido na progressão tumoral e, por isto, em piores desfechos ${ }^{22-24}$. Outros estudos mostram ainda que, estas alterações não desejadas na composição podem gerar desfechos clínicos oncológicos como, maior risco de recorrência da doença e progressão metastática subsequente ${ }^{50}$.

Do total, três estudos utilizaram densitometria por dupla emissão de raios-X para avaliação da composição corporal ${ }^{36-38}$. E dentre os três que encontraram relação entre esta e o estado menopausal, dois utilizaram este método ${ }^{36,37}$ e um utilizou tomografia computadorizada ${ }^{35}$. A densitometria por dupla emissão de raios-X avalia a composição corporal indiretamente e, é capaz de obter medidas de maior validade do que é possível usando métodos duplamente indiretos, como impedância bioelétrica e dobras cutâneas ${ }^{53}$.

A presente revisão integrativa mostrou falta de evidências consistentes em relação à associação entre o estado menopausal e a composição corporal em mulheres com CM. Isto possivelmente se deu pelo baixo número de artigos que atenderam aos critérios de elegibilidade e alguns aspectos metodológicos que impediram a comparação dos estudos, como maior percentual de pacientes na pós-menopausa; falta de informação quanto aos critérios utilizados para classificação do estado menopausal e de presença de grupo controle; grande heterogeneidade nos tamanhos amostrais, no momento clínico em que as pacientes foram investigadas e na forma de análise da composição corporal, o que provavelmente contribuiu para a inconsistência dos resultados observados. Ressalta-se, inclusive, a escassez de trabalhos comparando a relação entre composição corporal, estado menopausal e desfechos clínicos propriamente ditos entre estes indivíduos.

Por outro lado, destaca-se a inclusão de somente artigos com desenho longitudinal, evitando viés de causalidade reversa. Além do que, a maioria realizou ajustes de variáveis nas análises estatísticas, controlando potenciais fatores de confusão ou modificadores de efeito. Outrossim, ao que é conhecido, esta revisão inova em abordar a associação entre o estado menopausal e a composição corporal em mulheres com CM.

\section{Conclusão}

Dentre os estudos incluídos na presente revisão, quatro encontraram associação entre alterações na composição corporal e o estado menopausal de mulheres com CM. Um mostrou esta relação entre pacientes na pré-menopausa, enquanto que em outros dois, esta foi estatisticamente significativa na pós-menopausa. Em um deles, a sobrevida livre de doença a longo prazo foi significativamente pior no período pós-menopausa de pacientes com alta quantidade de gordura visceral. Por fim, outro relatou que as alterações foram similares entre ambos os estados menopausais.

Mesmo que os mecanismos teóricos implícitos na relação entre estado menopausal e composição corporal em mulheres com CM já sejam conhecidos, este cenário ainda precisa ser melhor estudado na prática, sobretudo no que condiz a desfechos clínicos. Por conseguinte, mais estudos futuros com metodologia robusta são necessários para clarificar a natureza dessa associação e os mecanismos biológicos envolvidos e como afetam, entre outras, a recorrência, o prognóstico e a mortalidade nesta população específica. Desta forma, fornecendo informações aos profissionais de saúde quanto à conduta adequada no que tange manejo terapêutico clínico e nutricional nesse setor crescente da população.

\section{Referências}

1. Aberoumandi SM, Mohammadhosseini M, Abasi E, Saghati S, Nikzamir N, Akbarzadeh A, et al. An update on applications of nanostructured drug delivery systems in cancer therapy: a review. Artificial Cells, Nanomedicine, and Biotechnology. 2016;45(6):1-11.

2. Li T, Mello-Thoms C, Brennan PC. Descriptive epidemiology of breast cancer in China: incidence, mortality, survival and prevalence. Breast Cancer Research and Treatment. 2016;159(3):395-406.

3. Pehlivan S, Kuzhan A, Yildirim Y, Fadiloglu C. Comfort and quality of life in patients with breast cancer undergoing radiation therapy. Journal of Balkan Union of Oncology. 2016;21(3):549-555

4. Brasil. Ministério da Saúde. Instituto Nacional de Câncer. Coordenação de Prevenção e Vigilância. Estimativa 2018: Incidência de Câncer no Brasil. Rio de Janeiro, RJ: INCA; 2018. 
5. Wu SG, Li H, Tang LY, Sun JY, Zhang WW, Li FY, et al. The effect of distant metastases sites on survival in de novo stage-IV breast cancer: A SEER database analysis. Tumor Biology. 2017;39(6):1-8.

6. Silva PA, Riul SS. Câncer de mama: fatores de risco e detecção precoce. Revista Brasileira de Enfermagem. 2011;64(6):1016-1021.

7. American Cancer Society. What causes cancer [Internet]? Atlanta [acesso em jan 2018]. Disponível em: <http:// www.cancer.org/cancer/cancercauses/>.

8. Anderson B, Flanigan J. Novel Methods for Measuring Global Cancer Burden Implications for Global Cancer Control. JAMA Oncology. 2015;1(4):425-427.

9. Chlebowski RT. Nutrition and physical activity influence on breast cancer incidence and outcome. The Breast. 2013;22(Suppl 2):S30-S37.

10. Tomasetti C, Vogelstein B. Variation in cancer risk among tissues can be explained by the number of stem cell divisions. Science. 2015;347(6217):78-81.

11. Shrivastava SR, Shrivastava PS, Ramasamy J. Exploring the role of dietary factors in the development of breast cancer. Journal of Cancer Research and Therapeutics. 2016;12(2):493-497.

12. Downs-Holmes C, Silverman, P. Breast cancer: Overview and updates. The Nurse Practitioner. 2011;36(12):20-26.

13. Friedenreich CM. Physical activity and breast cancer: Review of the epidemiologic evidence and biologic mechanisms. Recent Results in Cancer Research. 2011;188:125-139.

14. Hansen J, Stevens RG. Case-control study of shift-work and breast cancer risk in Danish nurses: Impact of shift systems. European Journal of Cancer. 2012;48(11):1722-1729.

15. Pratt MA. Paradoxical roles for FOXA1 in anti-estrogen resistance and as a luminal differentiation factor in breast cancer. Gland Surgery. 2012;1(1):9-11.

16. Harvey SL, Khasraw M. Alternative targeted therapy for early Her2 positive breast cancer. Gland Surgery. 2013;2(1):42-45.

17. American Cancer Society. How is breast cancer treated [Internet]? Atlanta [acesso em jan 2018]. Disponível em: $<$ http://www.cancer.org/cancer/breastcancer/detailedguide/breast-cancer-treating-general-info $>$.

18. Frenzel AP, Pastore CA, González MC. The influence of body composition on quality of life of patients with breast cancer. Nutrición Hospitalaria. 2013;28(5):1475-1482.

19. McDonald C, Bauer J, Capra S, Coll J. The muscle mass, omega-3, diet, exercise and lifestyle (MODEL) study - a randomised controlled trial for women who have completed breast cancer treatment. BMC Cancer. 2014;14(264):1-10.

20. Santilli V, Bernetti A, Mangone M, Paoloni M. Clinical definition of sarcopenia. Clinical Cases in Mineral and Bone Metabolism. 2014;11(3):177-180.
21. Anandavadivelan P, Brismar TB, Nilsson M, Johar AM, Martin L. Sarcopenic obesity: a probable risk factor for dose limiting toxicity during neo-adjuvant chemotherapy in oesophageal cancer patients. Clinical Nutrition. 2016;35(3):724-730.

22. Champ CE, Volek JS, Siglin J, Jin L, Simone NL. Weight gain, metabolic syndrome, and breast cancer recurrence: are dietary recommendations supported by the data? International Journal of Breast Cancer. 2012;2012(506868):1-9.

23. Cleary MP, Grossmann ME. Minireview: obesity and breast cancer: the estrogen connection. Endocrinology. 2009;150(6):2537-2542.

24. 24. Rock CL, Flatt SW, Laughlin GA, Gold EB, Thomson CA, Natarajan L. Reproductive steroid hormones and recurrence-free survival in women with a history of breast cancer. Cancer Epidemiology, Biomarkers and Prevention. 2008;17(3):614-620.

25. Bayar MA, Antoun S, Lanoy E. Statistical approaches for evaluating body composition markers in clinical cancer research. Expert Review of Anticancer Therapy. 2017;17(4):311-318.

26. Carneiro IP, Mazurak VC, Prado CM. Clinical implications of sarcopenic obesity in cancer. Current Oncology Reports. 2016;18(10):62.

27. Dieli-Conwright CM, Parmentier JH, Sami N, Lee K, Spicer D, Mack WJ, et al. Adipose tissue inflammation in breast cancer survivors: effects of a 16-week combined aerobic and resistance exercise training intervention. Breast Cancer Research and Treatment. 2018;168(1):147-157.

28. Moher D, Liberati A, Tetzlaff J, Altman DG. Preferred reporting items for systematic reviews and meta-analyses: the PRISMA statement. International Journal of Surgery. 2010;8(5):336-334.

29. Wells G, Shea B, O'Connell D, Peterson J, Welch V, Losos M, et al. The Newcastle-Ottawa Scale (NOS) for assessing the quality of nonrandomized studies in meta-analyses [Internet]. Ottawa: Ottawa Hospital Research Institute [acesso em mai 2018]. Disponível em: $<$ http://www.ohri.ca/programs/clinical_epidemiology/oxford.asp $>$.

30. Sterne JAC, Hernán MA, Reeves BC, Savović J, Berkman ND, Viswanathan M, et al. ROBINS-I: a tool for assessing risk of bias in non-randomised studies of interventions. BMJ. 2016;355:i4919.

31. Brasil. Ministério da Saúde. Secretaria de Ciência, Tecnologia e Insumos Estratégicos. Departamento de Ciência e Tecnologia. Diretrizes Metodológicas: elaboração de revisão sistemática e meta-análise de estudos observacionais comparativos sobre fatores de risco e prognóstico [Internet]. Brasília, DF: Ministério da Saúde; 2014 [acesso em mai 2018]. Disponível em: <http://bvsms.saude. gov.br/bvs/ct/PDF/diretrizes_metodologias_estudos_observacionais.pdf> 
32. Sterne JAC, Higgins JPT, Elbers RG, Reeves BC and the development group for ROBINS-I. Risk Of Bias In Non-randomized Studies of Interventions (ROBINS-I): detailed guidance [Internet]. 2016 [acesso em mai 2018]. Disponível em: <http://www.riskofbias.info/welcome/home >.

33. Akyol M, Alacacioglu A, Demir L, Kucukzeybek Y, Yildiz Y, Gumus Z, et al. The alterations of serum FGF-21 levels, metabolic and body composition in early breast cancer patients receiving adjuvante endocrine therapy. Cancer Biomarkers. 2017;18(4):441-449.

34. Dieli-Conwright CM, Wong L, Waliany S, Bernstein L, Salehian B, Mortimer JE. An Observational Study to Examine Changes in Metabolic Syndrome Components in Breast Cancer Patients Receiving Neoadjuvant or Adjuvant Chemotherapy. Cancer. 2016;122(17):2646-2653.

35. Iwase T, Sangai T, Nagashima T, Sakakibara M, Sakakibara J, Hayama S, et al. Impact of body fat distribution on neoadjuvant chemotherapy outcomes in advanced breast cancer patients. Cancer Medicine. 2016;5(1):41-48.

36. Cisneros KM, Romero JE, Torres AGG, Valencia ME, Estrada ROM, Ortiz OT, et al. Impacto del tratamiento antineoplásico en el estado nutricional en pacientes con cáncer de mama. Nutrición Hospitalaria. 2014;30(4):876-882.

37. Villaseñor A, Ballard-Barbash R, Baumgartner K, Baumgartner R, Bernstein L, McTiernan A, et al. Prevalence and prognostic effect of sarcopenia in breast cancer survivors: the HEAL Study. Journal of Cancer Survivorship. 2012;6(4):398-406.

38. Irwin ML, McTiernan A, Baumgartner RN, Baumgartner $\mathrm{KB}$, Bernstein L, Gilliland FD, et al. Changes in body fat and weight after a breast cancer diagnosis: Influence of demographic, prognostic and lifestyle factors. Journal of Clinical Oncology. 2005;23(4):774-782.

39. Sheean PM, Hoskins K, Stolley M. Body composition changes in females treated for breast cancer: a review of the evidence. Breast Cancer Research and Treatment. 2012;135(3):663-680.

40. Gordon AM, Hurwitz S, Shapiro CL, LeBoff MS. Premature ovarian failure and body composition changes with adjuvant chemotherapy for breast cancer. Menopause. 2011;18(11):1244-1248.

41. Nissen MJ, Shapiro A, Swenson KK. Changes in weight and body composition in women receiving chemotherapy for breast cancer. Clinical Breast Cancer. 2011;11(1):52-60.

42. Vance V, Mourtzakis M, McCargar L, Hanning R. Weight gain in breast cancer survivors: prevalence, pattern and health consequences. Obesity Reviews. 2011;12(4):282-294.

43. Rock CL, Demark-Wahnefried W. Nutrition and survival after the diagnosis of breast cancer: a review of the evidence. Journal of Clinical Oncology. 2002;20(15):3302-3316.
44. Di Sebastiano KM, Mourtzakis M. A critical evaluation of body composition modalities used to assess adipose and skeletal muscle tissue in cancer. Applied Physiology, Nutrition, and Metabolism. 2012;37(5):811-821.

45. Demark-Wahnefried W, Peterson BL, Winer EP, Marks L, Aziz N, Marcom PK, et al. Changes in weight, body composition, and factors influencing energy balance among premenopausal breast cancer patients receiving adjuvant chemotherapy. Journal of Clinical Oncology. 2001;19(9):2381-2389.

46. Walshe JM, Denduluri N, Swain SM. Amenorrhea in premenopausal women after adjuvant chemotherapy for breast cancer. Journal of Clinical Oncology. 2006;24(36):5769-5779.

47. Wang Q, Hassager C, Ravn P, Wang S, Christiansen C. Total and regional body-composition changes in early postmenopausal women: age-related or menopause-related? The American Journal of Clinical Nutrition. 1994;60(6):843-848.

48. Maltais ML, Desroches J, Dionne IJ. Changes in muscle mass and strength after menopause. Journal of Musculoskeletal and Neuronal Interactions. 2009;9(4):186-197.

49. Barbat-Artigas S, Aubertin-Leheudre M. Menopausal transition and fat distribution. Menopause. 2013;20(4):370-371.

50. Demark-Wahnefried W, Platz EA, Ligibel JA, Blair CK, Courneya KS, Meyerhardt JA, et al. The role of obesity in cancer survival and recurrence. Cancer Epidemiology, Biomarkers e Prevention. 2012;21(8):1244-1259.

51. Messier V, Rabasa-Lhoret R, Barbat-Artigas S, Elishaa B, Karelis AD, Aubertin-Leheudrec M. Menopause and sarcopenia: a potential role for sex hormones. Maturitas. 2011;68(4):331-336.

52. Jubrias SA, Odderson IR, Esselman PC, Conley KE. Decline in isokinetic force with age: muscle cross-sectional area and specific force. Pflügers Archiv - European Journal of Physiology. 1997;434(3):246-253.

53. Costa CS, Del-Ponte B, Assunção MCF, Santos IS. Consumption of ultra-processed foods and body fat during childhood and adolescence: a systematic review. Public Health Nutrition. 2017;21(1):148-159.

Como citar este artigo:

Poltronieri TS, Liberali R. Composição corporal e estado menopausal em mulheres com câncer de mama: uma revisão da literatura. Rev. Aten. Saúde. 2018;16(57):118-128. 
Anexo

Tabela 1 - Principais características e resultados das associações encontradas pelos estudos incluídos na presente revisão.

\begin{tabular}{|c|c|c|c|c|c|c|c|}
\hline Estudo/Local & $\begin{array}{c}\text { Avaliação da } \\
\text { qualidade }\end{array}$ & Desenho & População & $\begin{array}{l}\text { Método e frequ- } \\
\text { ência de avaliação } \\
\text { da CC }\end{array}$ & $\begin{array}{c}\text { Tempo de } \\
\text { seguimento }\end{array}$ & Resultados & Variáveis ajustadas \\
\hline $\begin{array}{l}\text { Akiol et al., } 2017 \\
\qquad[33]\end{array}$ & $3 / 6$ & $\begin{array}{l}\text { Longitudinal } \\
\text { (prospectivo) }\end{array}$ & $\begin{array}{l}72 \text { pacientes pré e pós-menopausa } \\
\text { com CM estadiamento I-III, RH } \\
\text { positivo, em uso de TMX (pré-me- } \\
\text { nopausa) ou AI (pós-menopausa), } \\
\text { previamente submetidas à cirurgia e } \\
\text { RXT adjuvante. } \\
51 \text { indivíduos saudáveis participaram } \\
\text { como grupo controle. }\end{array}$ & $\begin{array}{l}\text { BIA } \\
\text { No início do } \\
\text { estudo (basal) e } 12 \\
\text { meses depois. }\end{array}$ & 1 ano. & $\begin{array}{l}\text { Alterações na CC foram similares entre o gru- } \\
\text { po com TMX e o grupo com AI (não fornece } \\
\text { os resultados numéricos de cada grupo). }\end{array}$ & Não informa. \\
\hline $\begin{array}{l}\text { Dieli-Conwright } \\
\text { et al., 2016 } \\
\quad \text { [34] } \\
\text { Estados Unidos }\end{array}$ & $6 / 6$ & $\begin{array}{l}\text { Longitudinal } \\
\text { (prospectivo) }\end{array}$ & $\begin{array}{c}86 \text { mulheres na pré e pós-menopausa } \\
\text { com diagnóstico recente de CM em } \\
\text { estadiamento I-III em QT adjuvante } \\
\text { ou neoadjuvante }\end{array}$ & $\begin{array}{l}\text { No início do } \\
\text { estudo e uma } \\
\text { semana após a } \\
\text { conclusão do } \\
\text { protocolo de QT. }\end{array}$ & 3 anos. & $\begin{array}{l}\text { Aumento na quantidade de GC e o \%GC } \\
\text { foram significativamente maiores (aumento } \\
\text { de } 8,9 \% \text { na GC) depois da QT ( } \mathrm{p}<0,01) \text {. } \\
\text { A MM não mudou significativamente } \\
\text { ( } p>0.05) \text {. } \\
\text { Não foram observadas diferenças significati- } \\
\text { vas entre pacientes na pré e pós-menopausa } \\
\text { ( } p>0,01) \text {. }\end{array}$ & $\begin{array}{l}\text { Idade, raça e tipo/duração } \\
\text { da QT. }\end{array}$ \\
\hline
\end{tabular}

\begin{tabular}{|c|c|c|c|c|c|c|c|}
\hline $\begin{array}{l}\text { Iwase et al., } 2016 \\
\text { [35] } \\
\text { Japão }\end{array}$ & $3 / 6$ & $\begin{array}{c}\text { Longitudinal } \\
\text { (retrospectivo) }\end{array}$ & $\begin{array}{l}172 \text { pacientes com CM estadiamento } \\
\text { II-IV submetidas à cirurgia após } \\
\text { realização de QT neoadjuvante }\end{array}$ & $\begin{array}{l}\text { CT } \\
\text { Antes da QT } \\
\text { neoadjuvante }\end{array}$ & 1638 dias & $\begin{array}{l}\text { SFA e VFA aumentaram significativamente, } \\
\text { enquanto a LSMI diminuiu significativamente } \\
\text { após a menopausa ( } \mathrm{p}<0,05 \text { para ambos). } \\
\text { Razão VFA/SFA aumentou significativamente } \\
\text { após a menopausa }(\mathrm{p}<0,05) \text {. } \\
\text { Razão L/S não foi influenciada pelo estado } \\
\text { menopausal (não apresentou p-valor). } \\
\text { DDFS não diferiu significativamente } \\
\text { entre pacientes na pré-menopausa com } \\
\text { alta ou baixa VFA ( } \mathrm{p}=0,082 \text { ) } \\
\text { DDFS foi significativamente pior em pa- } \\
\text { cientes na pós-menopausa com alta VFA } \\
\text { comparado com aqueles com baixa (HR: } \\
2,79 \text {, IC } 95 \%: 1,29-6,05, \mathrm{p}<0,05 \text { ). }\end{array}$ & Não informa. \\
\hline $\begin{array}{c}\text { Cisneros et al., } \\
2014 \\
{[36]}\end{array}$ & $\begin{array}{l}\text { Risco mode- } \\
\text { rado de viés }\end{array}$ & $\begin{array}{l}\text { Quase experi- } \\
\text { mental }\end{array}$ & $\begin{array}{c}40 \text { mulheres diagnosticadas com CM } \\
\text { estadiamento I-III antes do início } \\
\text { de QT. }\end{array}$ & $\begin{array}{c}\text { Antes e depois } \\
\text { do tratamento } \\
\text { antineoplásico (6 } \\
\text { meses). }\end{array}$ & 6 meses & $\begin{array}{l}\text { Ao avaliar o efeito da QT sobre a CC, } \\
\text { encontrou-se um coeficiente de regressão de } \\
2,34(1,31-3,37), p=0,001 \text { para pacientes na } \\
\text { pré-menoupausa (ganho de em média } 2,3 \mathrm{~kg} \\
\text { de tecido adiposo durante o tratamento). } \\
\text { Já para pacientes na pós-menopausa, este } \\
\text { achado não foi significativo, } \mathrm{p}=0,227 .\end{array}$ & $\begin{array}{l}\text { Modelos que avaliaram } \\
\text { CC: ajuste para idade, } \\
\text { estado menopausal e } \\
\text { estadiamento. }\end{array}$ \\
\hline $\begin{array}{l}\text { Villaseñor et al., } \\
2012 \\
{[37]}\end{array}$ & $5 / 6$ & $\begin{array}{l}\text { Longitudinal } \\
\text { (coorte pros- } \\
\text { pectiva) }\end{array}$ & $\begin{array}{l}471 \text { mulheres sobreviventes de CM } \\
\text { do tipo invasivo, estadiamento I-III, } \\
\text { após tratamento oncológico poden- } \\
\text { do incluir cirurgia, QT e RXT. }\end{array}$ & $\begin{array}{l}\text { DEXA } \\
\begin{array}{l}\text { Na entrada do } \\
\text { estudo }\end{array}\end{array}$ & $\begin{array}{l}\text { Mediana de } 9,2 \\
\text { anos }\end{array}$ & $\begin{array}{l}\text { Sarcopenia foi mais comum em mulheres na } \\
\text { pós-menopausa }(\mathrm{p}=0,001) \text {. }\end{array}$ & $\begin{array}{l}\text { Todos os modelos: idade, } \\
\text { etnia/local de estudo, } \\
\text { circunferência da cintura, } \\
\text { \%GC, atividade física, está- } \\
\text { gio do CM no diagnóstico } \\
\text { e tipo de tratamento. } \\
\text { Modelos de mortalidade } \\
\text { para CM: adicionalmente } \\
\text { ajustados para o uso de } \\
\text { TMX. } \\
\text { Testadas interações } \\
\text { adicionando à análise o } \\
\text { estado sarcopênico, IMC, } \\
\text { estado menopausal e TMX } \\
\text { adjuvante. }\end{array}$ \\
\hline $\begin{array}{l}\text { Irwin et al., } 2005 \\
\qquad[38]\end{array}$ & $5 / 6$ & $\begin{array}{l}\text { Longitudinal } \\
\text { (coorte pros- } \\
\text { pectiva) }\end{array}$ & $\begin{array}{l}132 \text { mulheres sobreviventes de } \\
\text { CM, estadiamento in situ-III, após } \\
\text { tratamento oncológico podendo } \\
\text { incluir cirurgia, QT, RXT e uso ou } \\
\text { não de hormonioterapia (TMX) no } \\
\text { momento do estudo. }\end{array}$ & $\begin{array}{l}\text { Na entrada do } \\
\text { estudo (primeiro } \\
\text { ano pós-diagnós- } \\
\text { tico) e dois anos } \\
\text { após (terceiro ano } \\
\text { pós-diagnóstico). }\end{array}$ & 3 anos & $\begin{array}{l}\text { Não foram encontradas associações significa- } \\
\text { tivas entre mudanças na CC e estado meno- } \\
\text { pausal (não fornece p-valor). }\end{array}$ & $\begin{array}{l}\text { Modelos para avaliação } \\
\text { do \% de GC: ajuste para } \\
\text { estadiamento, tratamento, } \\
\text { local do estudo, tabagismo, } \\
\text { IMC, uso de TMX, idade, } \\
\text { estado menopausal, mu- } \\
\text { dança na atividade física } \\
\text { total, mudança na ingestão } \\
\text { calórica total, tempo entre } \\
\text { o diagnóstico e a entre- } \\
\text { vista inicial, meses após a } \\
\text { conclusão do tratamento, } \\
\text { etnia, educação, tratamento } \\
\text { completo, história familiar } \\
\text { de CM, história familiar de } \\
\text { diabetes tipo 2, diagnóstico } \\
\text { de diabetes tipo } 2 \text { e por } \\
\text { DCV. }\end{array}$ \\
\hline
\end{tabular}

F-21 - fator de crescimento de fibroblastos 21; CC - composição corporal; TMX - tamoxifeno; AI - inibidores da aromatase; VFA - área de gordura visceral; SFA - área de gordura subcutânea; LSMI - índice de músculo esquelético lombar; L/S - razão fígado/baço (marcador de gordura interna dos órgãos); DDFS - sobrevida livre de doença a longo prazo; MM - massa muscular; GC - gordura corporal; TC tomografia computadorizada; IMC - Índice de massa muscular; BIA - impedância bioelétrica; DEXA - densitometria por dupla emissão de raios-X; QT - quimioterapia; RH - receptor hormonal; DCV - doença cardiovascular. 